\title{
Analytic solutions for free vibration analysis of laminated beams in three-dimensional statement
}

\author{
Sergey Golushko ${ }^{1,2, *}$, Gleb Gorynin ${ }^{3}$, and Arseniy Gorynin ${ }^{1}$ \\ ${ }^{1}$ Novosibirsk State University \\ ${ }^{2}$ Institute of Computational Technologies of SB RAS \\ ${ }^{3}$ Surgut State University
}

\begin{abstract}
In this research we consider free vibrations of laminated beams in terms of three-dimensional linear theory of elasticity. Analytic solutions for natural frequencies of laminated beams are obtained by using an asymptotic splitting method. The results were compared with classical Euler"Bernoulli beam theory and Timoshenko beam theory.
\end{abstract}

\section{Introduction}

Let us consider a free vibrations of laminated beam (Fig. 1) with an arbitrary cross section constant along the length and symmetric about $O x$ axis. The beam consists of arbitrary number of isotropic layers. The layers are counted from top to bottom along $O x$ axis. The origin of coordinates is located in center of mass of cross section. Let denote number of layers by $s$, the layer number by $i$, the displacements along the $\mathrm{x}-, \mathrm{y}-$, and $\mathrm{z}$-axes by $u_{x}, u_{y}, u_{z}$, respectively and the length and height of the beam by $L$ and $h$.

We will use dimensionless variables and functions, keeping the original notation for simplicity:

$$
\begin{gathered}
\varepsilon=h / L, x \leftrightarrow \frac{x}{h}, y \leftrightarrow \frac{y}{h}, \quad z \leftrightarrow \frac{z}{h}, \quad u_{\alpha} \leftrightarrow \frac{u_{\alpha}}{\tilde{u}}, \lambda_{i} \leftrightarrow \frac{\lambda_{i}}{\tilde{E}} \\
\mu_{i} \leftrightarrow \frac{\mu_{i}}{\tilde{E}}, \quad \sigma_{\alpha \beta} \leftrightarrow \frac{\sigma_{\alpha \beta}}{\sigma_{0}}, \quad \sigma_{0}=\frac{\tilde{E} \tilde{u}}{h}, \quad \rho_{i} \leftrightarrow \frac{\rho_{i} \tilde{u} h}{\tilde{E} t_{0}^{2}}, \quad t \leftrightarrow \frac{t}{t_{0}}, \quad \omega_{n} \leftrightarrow \omega_{n} t_{0},
\end{gathered}
$$

where $\tilde{E}-$ is a characteristic value of Young ${ }^{\mathrm{TM}} \mathrm{S}$ modulus, $\tilde{u}-$ is a characteristic value for displacement $u_{x}, \sigma_{\alpha \beta}$ - are the components of the linear stress tensor, $\omega_{n}$ - is the natural frequency of the beam, $t_{0}$ - is characteristic time, $\rho_{i}, \mu_{i}, \lambda_{i}-$ are the density and the elastic constants of the material of the $i$-th layer. $\varepsilon-$ is a small parameter. Herein after we assume that : $\alpha \in(x, y, z), i=1, \ldots, s$.

The dimensionless equations of motion of a beam with no mass loads are:

$$
-\rho_{i} \frac{\partial^{2}\left(u_{\alpha}\right)_{i}}{\partial t^{2}}+\frac{\partial\left(\sigma_{\alpha x}\right)_{i}}{\partial x}+\frac{\partial\left(\sigma_{\alpha y}\right)_{i}}{\partial y}+\varepsilon \frac{\partial\left(\sigma_{\alpha z}\right)_{i}}{\partial z}=0 .
$$

\footnotetext{
*e-mail: s.k.golushko@gmail.com
} 


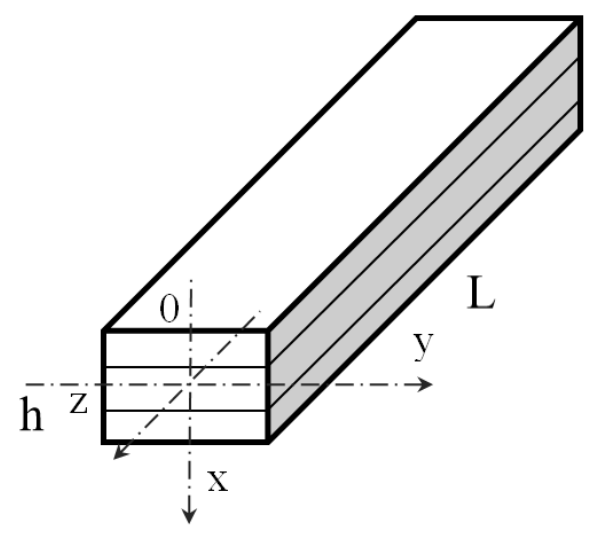

Figure 1. Laminated beam

Boundary conditions on the lateral surfaces of the beam (except for the ends):

$$
\left(\sigma_{\alpha x}\right)_{i} n_{x}+\left(\sigma_{\alpha y}\right)_{i} n_{y}=0
$$

The interfacial stresses $\left(\sigma_{\alpha n}\right)_{i}$ and displacements $\left(u_{\alpha}\right)_{i}$ are continuous:

$$
\left[\left(\sigma_{\alpha n}\right)\right]_{i}^{j}=0, \quad\left(\sigma_{\alpha n}\right)_{i}=\left(\sigma_{\alpha x}\right)_{i} n_{x}+\left(\sigma_{\alpha y}\right)_{i} n_{y}, \quad\left(u_{\alpha}\right)_{j}=\left(u_{\alpha}\right)_{i}
$$

The material of each layer obeys Hooke $\mathrm{TM}_{\mathrm{s}}$ law:

$$
\left(\sigma_{\alpha \beta}\right)_{i}=\lambda_{i} \theta \delta_{\alpha \beta}+2 \mu_{i} e_{\alpha \beta}, \quad \theta=\sum_{\gamma=1}^{3} e_{\gamma \gamma}, \lambda_{i}=\frac{v_{i} E_{i}}{\left(1-2 v_{i}\right)\left(1+v_{i}\right)}, \mu_{i}=\frac{E_{i}}{2\left(1+v_{i}\right)}
$$

where $e_{\alpha \beta}$ are the components of the linear strain tensor.

Cauchy equations for the linear strain tensor:

$$
\begin{gathered}
\left(e_{x x}\right)_{i}=\frac{\partial\left(u_{x}\right)_{i}}{\partial x},\left(e_{y y}\right)_{i}=\frac{\partial\left(u_{y}\right)_{i}}{\partial y},\left(e_{z z}\right)_{i}=\varepsilon \frac{\partial\left(u_{z}\right)_{i}}{\partial z},\left(e_{x y}\right)_{i}=\frac{1}{2}\left(\frac{\partial\left(u_{x}\right)_{i}}{\partial y}+\frac{\partial\left(u_{y}\right)_{i}}{\partial x}\right) \\
\left(e_{x z}\right)_{i}=\frac{1}{2}\left(\varepsilon \frac{\partial\left(u_{x}\right)_{i}}{\partial z}+\frac{\partial\left(u_{z}\right)_{i}}{\partial x}\right), \quad\left(e_{y z}\right)_{i}=\frac{1}{2}\left(\varepsilon \frac{\partial\left(u_{y}\right)_{i}}{\partial z}+\frac{\partial\left(u_{z}\right)_{i}}{\partial y}\right)
\end{gathered}
$$

Together formulas (2) - (6) represent a linear elasticity boundary-value problem in SaintVenant's statement.

For solving this problem method of asymptotic splitting is used [2]. The unknown displacement vector functions and stress tensor functions are approximated by following rules: 


$$
\begin{gathered}
\left(u_{\beta}\right)_{i}^{(n)}(\bar{r}, t, \varepsilon)=\sum_{k=0}^{n+1}\left(U_{\beta}\right)_{i}^{(2 k)} \frac{\partial^{2 k} u_{0}^{(n)}}{\partial z^{2 k}} \varepsilon^{2 k},\left(u_{z}\right)_{i}^{(n)}(\bar{r}, t, \varepsilon)=\sum_{k=0}^{n}\left(U_{z}\right)_{i}^{(2 k+1)} \frac{\partial^{2 k+1} u_{0}^{(n)}}{\partial z^{2 k+1}} \varepsilon^{2 k+1}, \\
\beta \in(x, y), \quad\left(U_{x}\right)_{i}^{(0)}=1, \quad\left(U_{y}\right)_{i}^{(0)}=0, \quad\left(U_{z}\right)_{i}^{(1)}=-\left(x-c_{0}\right), \\
\left(\sigma_{\alpha \beta}\right)_{i}^{(n)}(\bar{r}, t, \varepsilon)=\sum_{k=1}^{n+1}\left(\tau_{\alpha \beta}\right)_{i}^{(2 k)} \frac{\partial^{2 k} u_{0}^{(n)}}{\partial z^{2 k}} \varepsilon^{2 k},\left(\sigma_{z z}\right)_{i}^{(n)}(\bar{r}, t, \varepsilon)=\sum_{k=1}^{n}\left(\tau_{z z}\right)_{i}^{(2 k)} \frac{\partial^{2 k} u_{0}^{(n)}}{\partial z^{2 k}} \varepsilon^{2 k}, \\
\left(\sigma_{\alpha z}\right)_{i}^{(n)}(\bar{r}, t, \varepsilon)=\sum_{k=1}^{n}\left(\tau_{\alpha z}\right)_{i}^{(2 k+1)} \frac{\partial^{2 k+1} u_{0}^{(n)}}{\partial z^{2 k+1}} \varepsilon^{2 k+1}, \quad \alpha, \beta \in(x, y),
\end{gathered}
$$

where $\left(U_{\alpha}\right)_{i}^{(k)}$ are the characteristic functions of the displacement vector; $\left(u_{\alpha}\right)_{i}^{(n)}(\bar{r}, t, \varepsilon)$ are the displacement components of the $i$-th layer in the $n$-th order asymptotic approximation; $\left(\tau_{\alpha \beta}\right)_{i}^{(k)}(x, y)$ are the characteristic functions of the tensor stress field in the beam cross section; $u_{0}(z, t)$ is the deflection function. $\left(U_{\alpha}\right)_{i}^{(k)}$ :

Relations between characteristic functions $\left(\tau_{\alpha \beta}\right)_{i}^{(k)}(x, y)$ and characteristic functions

$$
\begin{aligned}
\left(\tau_{x y}\right)_{i}^{2 k}= & \mu_{i}\left(\frac{\partial\left(U_{y}\right)_{i}^{(2 k)}}{\partial x}+\frac{\partial\left(U_{x}\right)_{i}^{(2 k)}}{\partial y}\right), \quad\left(\tau_{z \alpha}\right)_{i}^{2 k+1}=\mu_{i}\left(\frac{\partial\left(U_{z}\right)_{i}^{(2 k+1)}}{\partial \alpha}+\left(U_{\alpha}\right)_{i}^{(2 k)}\right) \\
& \left(\tau_{z z}\right)_{i}^{2 k}=\left(\lambda_{i}+2 \mu_{i}\right)\left(U_{z}\right)_{i}^{(2 k-1)}+\lambda_{i}\left(\frac{\partial\left(U_{x}\right)_{i}^{(2 k)}}{\partial x}+\frac{\partial\left(U_{y}\right)_{i}^{(2 k)}}{\partial y}\right) \\
& \left(\tau_{x x}\right)_{i}^{2 k}=\left(\lambda_{i}+2 \mu_{i}\right) \frac{\partial\left(U_{x}\right)_{i}^{(2 k)}}{\partial x}+\lambda_{i}\left(\left(U_{z}\right)_{i}^{(2 k-1)}+\frac{\partial\left(U_{y}\right)_{i}^{(2 k)}}{\partial y}\right) \\
& \left(\tau_{y y}\right)_{i}^{2 k}=\left(\lambda_{i}+2 \mu_{i}\right) \frac{\partial\left(U_{y}\right)_{i}^{(2 k)}}{\partial y}+\lambda_{i}\left(\left(U_{z}\right)_{i}^{(2 k-1)}+\frac{\partial\left(U_{x}\right)_{i}^{(2 k)}}{\partial x}\right)
\end{aligned}
$$

In case of free vibrations transverse the distributed load is equal to zero. According to the approximation (7), equation of free vibrations looks as follows [3]:

$$
M \frac{\partial^{2} u_{0}^{(n)}}{\partial t^{2}}+\sum_{k=2}^{n+1} G_{x z}^{(2 k-1)} \frac{\partial^{2 k} u_{0}^{(n)}}{\partial z^{2 k}} \varepsilon^{2 k}=0, \quad G_{x z}^{(2 k-1)}=-\sum_{i=1}^{s} \int_{F_{i}}\left(\tau_{x z}\right)_{i}^{(2 k-1)} d F
$$

where $G_{x z}^{(2 k-1)}$ are the characteristic stiffnesses of the cross section; $\mathrm{M}$ is the dimensionless mass of the beam cross section per unit length. To find equation of free vibrations (9) it is necessary to determine characteristic stiffnesses by solving several boundary-value problems in the beam cross section.

\section{Boundary-value problems in the beam cross section}

Approximation of displacement vector and stress tensor of the form (7) allow us to split the original three-dimensional problem into the $(n+1)$ much easier two-dimensional problems, where the unknowns are characteristic functions $\left(\tau_{\alpha \beta}\right)_{i}^{(n)}$. The characteristic number $k$ changes from 1 to $n+1$. The first two boundary-value problems are described below.

System of equations for boundary-value problem 
with number $k=1$ :

$$
\left\{\begin{array}{l}
\frac{\partial\left(\tau_{\alpha x}\right)_{i}^{(2)}}{\partial x}+\frac{\partial\left(\tau_{\alpha y}\right)_{i}^{(2)}}{\partial y}=0 \\
\frac{\partial\left(\tau_{z x}\right)_{i}^{(3)}}{\partial x}+\frac{\partial\left(\tau_{z y}\right)_{i}^{(3)}}{\partial y}+\left(\tau_{z z}\right)_{i}^{(2)}=0
\end{array}\right.
$$

with number $k=2$ :

$$
\left\{\begin{array}{l}
\frac{\partial\left(\tau_{x x}\right)_{i}^{(4)}}{\partial x}+\frac{\partial\left(\tau_{x y}\right)_{i}^{(4)}}{\partial y}+\left(\tau_{x z}\right)_{i}^{(3)}+\rho_{i} \frac{G_{x z}^{(3)}}{M}=0 \\
\frac{\partial\left(\tau_{x y}\right)_{i}^{(4)}}{\partial x}+\frac{\partial\left(\tau_{y y}\right)_{i}^{(4)}}{\partial y}+\left(\tau_{y z}\right)_{i}^{(3)}=0 \\
\frac{\partial\left(\tau_{z x}\right)_{i}^{(5)}}{\partial x}+\frac{\partial\left(\tau_{z y}\right)_{i}^{(5)}}{\partial y}+\left(\tau_{z z}\right)_{i}^{(4)}-\rho_{i} \frac{G_{x z}^{(3)}}{M}\left(x-c_{0}\right)=0
\end{array}\right.
$$

Boundary conditions on the border of cross section for (10), (11):

$$
\left(\tau_{\alpha x}\right)_{i}^{(2 k)} n_{x}+\left(\tau_{\alpha y}\right)_{i}^{(2 k)} n_{y}=0, \quad\left(\tau_{z x}\right)_{i}^{(2 k+1)} n_{x}+\left(\tau_{z y}\right)_{i}^{(2 k+1)} n_{y}=0, \quad \alpha \in(x, y) .
$$

Conditions at the interface between layers for (10), (11):

$$
\begin{gathered}
\left(\tau_{n \beta}\right)_{i}^{(2 k)}=\left(\tau_{n \beta}\right)_{j}^{(2 k)}, \quad\left(\tau_{n z}\right)_{i}^{(2 k)}=\left(\tau_{n z}\right)_{j}^{(2 k)}, \\
\left(U_{\beta}\right)_{i}^{(2 k)}=\left(U_{\beta}\right)_{j}^{(2 k)}, \quad\left(U_{z}\right)_{i}^{(2 k+1)}=\left(U_{z}\right)_{j}^{(2 k+1)}, \alpha \in(x, y, z), \beta \in(x, y) .
\end{gathered}
$$

According to [2] approximation of displacement vector and stress tensor of the form (7) with consideration to (10)-(13) gives an asymptotic solution of linear elasticity problem (2)(6) with residual proportional to $\varepsilon^{(2 n+2)}$.

The following equalities are fulfilled [4]:

$$
\begin{gathered}
\left(\tau_{z z}\right)_{i}^{(2)}=v\left(\tau_{x x}\right)_{i}^{(2)}+v\left(\tau_{y y}\right)_{i}^{(2)}-E_{i}\left(x-c_{0}\right), \\
\left(\tau_{z z}\right)_{i}^{(4)}=v\left(\tau_{x x}\right)_{i}^{(4)}+v\left(\tau_{y y}\right)_{i}^{(4)}+E_{i}\left(U_{z}\right)_{i}^{(3)} .
\end{gathered}
$$

Equations (10), (11) with (12), (13) represent boundary-value problems in the beam cross section for $k=1,2$. In case of arbitrary cross section it is necessary to use numerical methods for solving corresponding boundary-value problems. However for widely used types of cross sections as a rectangular or I-beam cross sections it is possible to find an analytic solution.

\section{Solution for rectangular cross section}

From the equation of vibrations (9) follows the formula of natural frequencies for a hinged simple beam of an arbitrary cross section [3]:

$$
\begin{gathered}
\omega_{m}^{(n)}=\sqrt{\sum_{k=2}^{n+1} G_{x z}^{(2 k-1)}(\pi m \varepsilon)^{2 k}(-1)^{k},} \\
\omega_{m}^{(1)}=(\pi m \varepsilon)^{2} \sqrt{\frac{G_{x z}^{(3)}}{M}}, \quad \omega_{m}^{(2)}=\omega_{m}^{(1)} \sqrt{1-\frac{G_{x z}^{(5)}}{G_{x z}^{(3)}}(\pi m \varepsilon)^{2}},
\end{gathered}
$$

where $\omega_{m}^{(1)}$ and $\omega_{m}^{(2)}$ are the first and second approximations of natural frequency. 


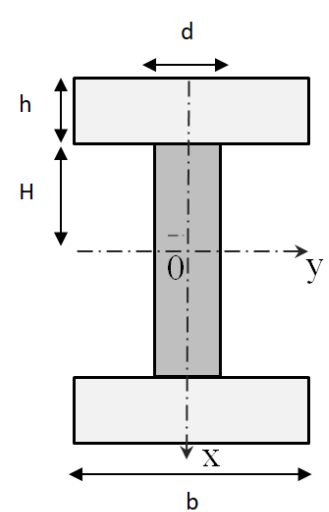

a)

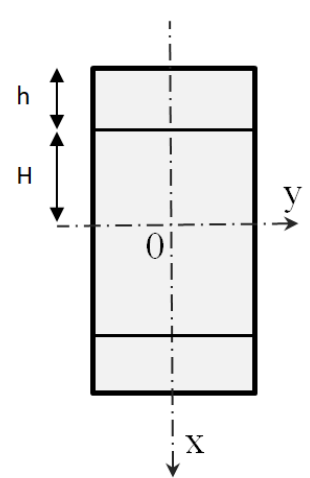

b)

Figure 2. ) - I-beam cross section; b) - rectangular cross section

To find $\omega_{m}^{(1)}, \omega_{m}^{(2)}$ it is necessary to solve first two boundary-value problems in the beam cross section for $k=1,2$ and determine characteristic stiffnesses $G_{x z}^{(3)}, G_{x z}^{(5)}$.

First boundary-value problem (10), (12), (13) for $k=1$ has an exact solution:

$$
\begin{gathered}
\left(\tau_{z z}\right)_{i}^{(2)}=-\left(x-c_{0}\right) E_{i}, \quad\left(\tau_{\alpha \alpha}\right)_{i}^{(2)}=0, \quad \alpha \in(x, y), \\
\left(U_{x}\right)_{i}^{(2)}=0.5\left(-v y^{2}+v\left(x-c_{0}\right)^{2}+C_{2}\right), \quad \int_{F}\left(U_{x}\right)_{i}^{(2)} d F=0,\left(U_{y}\right)_{i}^{(2)}=v y\left(x-c_{0}\right) .
\end{gathered}
$$

Constant $c_{0}$ need to be determined from condition:

$$
\int_{F_{i}}\left\langle\left(\tau_{z z}\right)_{i}^{(2)}\right\rangle d F=0
$$

For $G_{x z}^{(3)}$ with consideration to (16) the following formula is satisfied:

$$
G_{x z}^{(3)}=-\sum_{i=1}^{s} \int_{F_{i}}\left(\tau_{x z}\right)_{i}^{(3)} d F=-\sum_{i=1}^{s} \int_{F_{i}} x\left(\tau_{z z}\right)_{i}^{(2)} d F=[E I], \quad[E I]=\sum_{i=1}^{s} E_{i} I_{i}
$$

Formulas (16), (18) are satisfied for an arbitrary cross section. It means that solution of a first boundary-value problem does not depend on a form of cross section.

To find $G_{x z}^{(5)}$ we multiply the third equality $(11)$ by $\left(x-c_{0}\right)$ and integrate over the entire beam cross section:

$$
\begin{gathered}
\sum_{i=1}^{s} \int_{F_{i}}\left(x \frac{\partial\left(\tau_{x z}\right)_{i}^{(5)}}{\partial x}+x \frac{\partial\left(\tau_{y z}\right)_{i}^{(5)}}{\partial y}\right) d F-I_{z z}^{(4)}-\frac{G_{x z}^{(3)}}{M} \sum_{i=1}^{s} \int_{F_{i}} \rho_{i}\left(x-c_{o}\right)^{2} d F=0 \\
I_{z z}^{(4)}=-\sum_{i=1}^{s} \int_{F_{i}}\left(x-c_{0}\right)\left(\tau_{z z}\right)_{i}^{(4)} d F
\end{gathered}
$$

Introducing $x$ under the derivative sign and replacing the integral over cross section to the integral over the boundary of cross section, we obtain the formula:

$$
G_{x z}^{(5)}=I_{z z}^{(4)}+\frac{G_{x z}^{(3)}}{M}[\rho I], \quad[\rho I]=\sum_{i=1}^{s} \rho_{i} I_{i}=\sum_{i=1}^{s} \int_{F_{i}} \rho_{i}\left(x-c_{o}\right)^{2} d F .
$$


To determine $I_{z z}^{(4)}$ in formula (20) it is necessary to take into account the cross-sectional shape. To solve the subsequent boundary-value problems, we use the procedure of averaging the characteristic functions over the cross section. The sought stresses will vary slightly within the thin wall and it can be assumed that their average values will almost coincide with the values themselves. A similar procedure for solving boundary value problems is described in [5].

Here we present a solution for a thin-walled rectangular beams. The procedure of averaging the characteristic functions over the cross section looks as follows:

$$
\langle f\rangle=\frac{1}{b} \int_{-0.5 b}^{0.5 b} f(\alpha, s) d s .
$$

Apply the averaging procedure (21) to the second equation (11) to find unknown characteristic functions $\left(\tau_{z x}\right)_{i}^{(3)}\left(\tau_{z y}\right)_{i}^{(3)}$. We use the conditions on the lateral surface of the beam (11) and take into account the solution (16):

$$
\frac{d\left\langle\left(\tau_{z x}\right)_{i}^{(3)}\right\rangle}{d x}=x E_{i}, \quad\left\langle\left(\tau_{z x}\right)_{i}^{(3)}\right\rangle=\int_{-0.5}^{x} E_{i} \xi d \xi
$$

The function $\left(\tau_{z y}\right)_{i}^{(3)}$ is antisymmetric across the width of the cross section, therefore $\left(\tau_{z y}\right)_{i}^{(3)}=0$. We average the second equality (8) and substitute in it the averaged third expression from (16), integrate over the variable $x$ and express the averaged characteristic function $\left(U_{z}\right)_{i}^{(3)}$ :

$$
\left\langle\left(U_{z}\right)\right\rangle_{i}^{(3)}=\int_{-0.5}^{x}\left(\frac{\left\langle\left(\tau_{z x}\right)_{i}^{(3)}\right\rangle}{\mu_{i}}-\left\langle\left(U_{x}\right)_{i}^{(2)}\right\rangle\right) d \xi+C_{1}, \quad\left\langle\left(U_{x}\right)_{i}^{(2)}\right\rangle=0.5 v\left(x^{2}-\frac{1}{12}\right) .
$$

The constant $C_{1}$ in the expression (23) can be found from the equality:

$$
\int_{-0.5}^{0.5}\left\langle\left(\tau_{z z}\right)_{i}^{(4)}\right\rangle d x=0 .
$$

The expression (24) follows from the fact that with pure bending, the longitudinal force is zero.

We now proceed to the second boundary value problem. We average the first equation (11) and use the conditions at the cross section boundary (12):

$$
\frac{d\left(\left\langle\tau_{x x}\right)_{i}^{(4)}\right\rangle}{d x}+\left\langle\left(\tau_{z x}\right)_{i}^{(3)}\right\rangle+\frac{G_{x z}^{(3)}}{M}\left\langle\rho_{i}\right\rangle=0,\left.\quad\left\langle\left(\tau_{x x}\right)_{i}^{(4)}\right\rangle\right|_{x=-0.5}=0 .
$$

We integrate (25) and get:

$$
\left\langle\left(\tau_{x x}\right)_{i}^{(4)}\right\rangle=-\int_{-0.5}^{x}\left(\left\langle\left(\tau_{z x}\right)_{i}^{(3)}\right\rangle d \xi-\frac{G_{x z}^{(3)}}{M} \int_{-0.5}^{x}\left\langle\rho_{i}\right\rangle d \xi .\right.
$$

Due to antisymmetry, the average values of the following functions are zero:

$$
\left\langle\left(\tau_{y y}\right)_{i}^{(4)}\right\rangle=0, \quad\left\langle\left(\tau_{x y}\right)_{i}^{(4)}\right\rangle=0 .
$$

We average the second expression over the width of the cross section (14):

$$
\left\langle\left(\tau_{z z}\right)_{i}^{(4)}\right\rangle=v\left\langle\left(\tau_{x x}\right)_{i}^{(4)}\right\rangle+E_{i}\left\langle\left(U_{z}\right)_{i}^{(3)}\right\rangle .
$$

Thus, the characteristic stiffnesses are needed to calculate the first and the second approximations of natural frequency (15) can be found analytically by the formulas (18), (20), (22), (23), (24), (26), (28). 


\section{Analysis of the solution}

To compare the obtained results, we consider two theories of free vibrations of a homogeneous beam: the classical Euler"Bernoulli beam theory and Timoshenko beam theory. The difference between Timoshenko ${ }^{\mathrm{TM}}$ s theory is that it takes into account the deplanation and inertia of the rotation of the cross section during vibrations [1]. The equation of free vibrations of a homogeneous beam in Timoshenko ${ }^{\mathrm{TM}} \mathrm{S}$ theory is as follows [6]:

$$
\frac{E I}{\rho F} \frac{\partial^{4} u_{0}}{\partial z^{4}}+\frac{\partial^{2} u_{0}}{\partial t^{2}}-\frac{I}{F}\left(1+\frac{E}{k^{\prime} G}\right) \frac{\partial^{4} u_{0}}{\partial z^{2} t^{2}}+\frac{\rho I}{k^{\prime} G F} \frac{\partial^{4} u_{0}}{\partial t^{4}}=0, \quad k^{\prime}=\frac{10(1+v)}{12+11 v},
$$

where $G$ is a shear modulus; $k^{\prime}$ is a coefficient depending on the shape of the cross section. The formula for calculating the natural frequencies of a hinged simple beam::

$$
\omega_{m}^{T}=a \frac{\pi^{2} m^{2}}{L^{2}}\left[1-\frac{1}{2} \frac{I \pi^{2} m^{2}}{F L^{2}}\left(1+\frac{E}{k^{\prime} G}\right)\right], \quad a=\sqrt{\frac{E I}{\rho F}} .
$$

The first and the second approximations of natural frequency (15) in dimensional variables are:

$$
\omega_{m}^{(1)}=[a] \frac{\pi^{2} m^{2}}{L^{2}}, \quad \omega_{m}^{(2)}=[a] \frac{\pi^{2} m^{2}}{L^{2}} \sqrt{1-\zeta \frac{\pi^{2} m^{2} h^{2}}{L^{2}}}, \quad[a]=\sqrt{\frac{[E I]}{[\rho F]}}, \quad \zeta=\frac{G_{x z}^{(5)}}{[E I]} .
$$

In case of homogeneous beam the frequency $\omega_{m}^{(1)}$ is equal to the frequency predicted by classical beam theory based on the Bernoulli hypothesis. The frequency $\omega_{m}^{(2)}$ takes into account the deplanation and inertia of the rotation of the cross section.

Let us compare two formulas (31) and (30) in case of homogeneous beam. To do that, we introduce relative differences $\Delta_{m}^{\omega}$ between natural frequencies:

$$
\Delta_{m}^{\omega(2) T}=\frac{\left(\omega_{m}^{(2)}-\omega_{m}^{(T)}\right)}{\omega_{m}^{(2)}}, \quad \Delta_{m}^{\omega(12)}=\frac{\left(\omega_{m}^{(1)}-\omega_{m}^{(2)}\right)}{\omega_{m}^{(2)}}, \quad \varepsilon_{h}=\frac{b}{h}
$$

where $\varepsilon_{h} \ll 1$ is a ratio between width and height of the cross section. It is important to note that the formulas (31) are applicable as long as the condition is true:

$$
(\pi m \varepsilon)<1
$$

The expression (33) imposes a restriction on the length of the beam and the ordinal number of the frequency.

Table 1. Relative differences for homogeneous beam, $v=0.25$

\begin{tabular}{|c|c||c|c|c|c|c|c|}
$\varepsilon_{h}$ & $\varepsilon$ & $\Delta_{1}^{\omega T(2)}$ & $\Delta_{2}^{\omega T(2)}$ & $\Delta_{3}^{\omega T(2)}$ & $\Delta_{1}^{\omega(12)}$ & $\Delta_{2}^{\omega(12)}$ & $\Delta_{3}^{\omega(12)}$ \\
\hline \multirow{4}{*}{0.2} & $1 / 15$ & 0.00006 & -0.00005 & -0.0015 & 0.0072 & 0.03 & 0.071 \\
& $1 / 10$ & 0.00007 & -0.0015 & -0.012 & 0.0164 & 0.071 & 0.186 \\
& $1 / 7$ & -0.00014 & -0.0001 & -0.094 & 0.0344 & 0.164 & 0.56 \\
\hline
\end{tabular}

From Table 1, we can see that the relative difference for the first frequencies does not exceed $1 \%$. As the $\varepsilon$ parameter increases, the relative difference increases to $19 \%$ and $56 \%$ with $\varepsilon=1 / 7$. The deviation of $56 \%$ is not reliable, since in this case the condition (33) is not satisfied. The results obtained weakly depend on $v$ and do not depend on $\varepsilon_{h}$. 
Table 2. Relative differences for three-layer beam, $v=0.25, \varepsilon=1 / 15$

\begin{tabular}{|c|c||c|c|c|}
$E_{e x} ; E_{c} ;$ & $H ; h ;$ & $\Delta_{1}^{\omega(12)}$ & $\Delta_{2}^{\omega(12)}$ & $\Delta_{3}^{\omega(12)}$ \\
\hline$E_{e x}=10, E_{c}=1$ & $H=0.45, h=0.05$ & 0.02 & 0.087 & 0.237 \\
$E_{e x}=10, E_{c}=1$ & $H=0.4, h=0.1$ & 0.029 & 0.136 & 0.424 \\
$E_{e x}=5, E_{c}=1$ & $H=0.45, h=0.05$ & 0.013 & 0.054 & 0.138 \\
$E_{e x}=5, E_{c}=1$ & $H=0.4, h=0.1$ & 0.017 & 0.075 & 0.197 \\
$E_{e x}=10, E_{c}=1$ & $H=0.3, h=0.2$ & 0.039 & 0.189 & 0.715 \\
$E_{e x}=100, E_{c}=1$ & $H=0.45, h=0.05$ & 0.161 & - & - \\
\hline
\end{tabular}

In Table 2 the results for a three-layer beam consisting of isotropic materials are presented. It is assumed that the Young's modulus of the outer layers is larger than in the middle layer $\left(E_{e x}>E_{c}\right)$ and Poisson's coefficients of the layers are equal. The thickness of the layers varies. It can be seen that the more different the mechanical characteristics of the layers, the higher the relative differences. For a three-layer beam, in which the Young's modules differ 100 times, the relative error for the first natural frequency reaches $16 \%$. Thus, the use of second-order asymptotic approximations and higher is more justified for non-homogeneous beams.

A situation may arise when the magnitude under the root in the formula (15) becomes negative. In this case, the lower bound for the true frequency will be zero. In such cases, there is a dash in the table.

\subsection{Solution for I-beam cross section}

Similar to a beam of rectangular section, an I-beam was calculated. The averaging of characteristic functions was carried out over the wall width $d$ and over the height of the shelves $h$.

In Table 3 the relative differences of natural frequencies for a homogeneous I-beam are presented. It can be seen that the natural frequencies predicted by the second asymptotic approximation are almost equal to the ones predicted by Timoshenko theory. The difference between them is small and does not exceed 3\%.

Here we present the results for a three-layer non-homogeneous I-beam (Table 4), in which the wall is made of one material and the shelves are of another. The effect of cross section deplanation in this case increases. When the Young's modulus differs 10 times, the relative differences for the first frequency reaches $25 \%$.

\section{Conclusions}

The asymptotic splitting method allows to obtain the first approximations of the natural frequencies $\omega_{m}^{(n)}$ analytically when the Poisson's coefficients in each layer are equal. The first approximation $\omega_{m}^{(1)}$ corresponds to the frequencies predicted by the classical theory of the Bernoulli-Euler beam and estimate the true frequency from above. The second approximation $\omega_{m}^{(2)}$ takes into account the deplanation and inertia of the rotation of the cross section during vibrations and evaluates the true frequency from below. For a hinged simple homogeneous beam, the second approximation $\omega_{m}^{(2)}$ almost completely coincides with the solution obtained from the refined Timoshenko theory.

For multi-layer beams, the effect of shear and inertia of rotation increases with the heterogeneity of the beam. Except for cases when the Young's moduli of the layers differ significantly $\left(E_{e x} / E_{c}>100\right)$, the influence of the shear and inertia of the rotation of the cross 
Table 3. Relative differences for homogeneous I-beam, $v=0.25, E_{n}=E_{c}$

\begin{tabular}{|c|c||c|c|c|}
$\varepsilon$ & $H, h, d, b$ & $\Delta_{1}^{\omega(2 T)}$ & $\Delta_{2}^{\omega(2 T)}$ & $\Delta_{3}^{\omega(2 T)}$ \\
\hline \multirow{5}{*}{$\varepsilon=1 / 15$} & $H=0.8, h=0.1, d=0.1, b=0.7$ & 0.002 & 0.006 & 0.003 \\
& $H=0.9, h=0.05, d=0.05, b=0.7$ & 0.001 & 0.001 & -0.03 \\
& $H=0.8, h=0.1, d=0.1, b=0.5$ & -0.003 & -0.004 & -0.02 \\
& $H=0.8, h=0.1, d=0.1, b=0.5$ & 0.005 & 0.018 & 0.034 \\
\hline \multirow{5}{*}{$\varepsilon=1 / 7$} & $H=0.8, h=0.1, d=0.1, b=0.7$ & 0.006 & -0.28 & - \\
& $H=0.9, h=0.05, d=0.05, b=0.7$ & -0.0002 & -1.1 & - \\
& $H=0.8, h=0.1, d=0.1, b=0.5$ & -0.005 & -0.22 & - \\
& $H=0.8, h=0.1, d=0.1, b=0.5$ & 0.02 & 0.016 & - \\
\hline
\end{tabular}

Table 4. Relative differences for non-homogeneous I-beam, $v=0.25, \varepsilon=1 / 15$

\begin{tabular}{|c|c||c|c|c|}
$E_{e x}, E_{c}$ & $H, h, d, b$ & $\Delta_{1}^{\omega(12)}$ & $\Delta_{2}^{\omega(12)}$ & $\Delta_{3}^{\omega(12)}$ \\
\hline$E_{e x}=E_{c}=1$ & $H=0.9, h=0.05, d=0.05, b=0.7$ & 0.027 & 0.129 & 0.364 \\
$E_{e x}=E_{c}=1$ & $H=0.8, h=0.1, d=0.1, b=0.7$ & 0.023 & 0.103 & 0.29 \\
$E_{e x}=5, E_{c}=1$ & $H=0.8, h=0.1, d=0.1, b=0.7$ & 0.067 & 0.39 & - \\
$E_{e x}=10, E_{c}=1$ & $H=0.9, h=0.05, d=0.05, b=0.7$ & 0.25 & - & - \\
$E_{e x}=10, E_{c}=1$ & $H=0.8, h=0.1, d=0.1, b=0.7$ & 0.212 & - & - \\
$E_{e x}=100, E_{c}=1$ & $H=0.9, h=0.05, d=0.05, b=0.7$ & - & - & - \\
\hline
\end{tabular}

section is not large and the classical theory can be used with an acceptable accuracy. The insignificant difference in the results can be explained by the fact that for isotropic materials the effect of transverse deformations is small.

The research was supported financially by Russian Foundation for Basic Research, Project 18-29-18029

\section{References}

[1] S. P. Timoshenko, W. Weaver Jr. , D. H. Young Vibration Problems in Engineering // John Wiley Sons, 1990 - pp. 624.

[2] Gorynin G.L., Nemirovsky Yu.V. Spatial tasks of bending and torsion of layered structures. Asymptotic splitting method. - Novosibirsk, 2004. - 409 p. (in Russian)

[3] Gorynin G.L., Nemirovsky Yu.V. Tranverse vibration of laminated beams in ThreeDimensional Formulation // Int. Appl. Mech. - 2005. - 41, N. 6. - P. 631-645.

[4] Gorynin G.L., Nemirovsky Yu.V. Deformation of laminated anisotropic bars in the three-dimensional statement 1.Transverse-longitudinal bending and edge compatibility condition // Mechanics of Composite Materials, Vol. 45, N. 3, 2009. - pp. 257-280.

[5] Gorynin G.L., Gorynina O.G. The study of the stress-strain state of a three-layer Ibeam in three-dimensional statement // Vestnik Sibadi. - 2012. - N. 5 (27). - pp. 49-54. (in Russian)

[6] J. M. Gere, S. P. Timoshenko Mechanics of materials. 4th Edition, PWS, 1997. - 912 p. 